\title{
Descripción clínica y epidemiológica de un grave brote de salmonelosis transmitida por alimentos
}

\author{
Diego García-Huidobro, Mónica Carreño, Sergio Alcayaga y Jenny Ulloa
}

\begin{abstract}
Pontificia Universidad Católica de Chile, Santiago. Departamento de Medicina Familiar (DGH).

Departamento de Pediatría (MC). Secretaría Regional Ministerial de Salud, Región Metropolitana. Departamento de Salud Pública Sub-Departamento de Epidemiología (SA, JU).

Recibido: 11 de mayo de 2011

Aceptado: 9 de enero de 2012

Este trabajo no contempla fuentes de financiamiento.

Los autores no presentan conflictos de interés.

Correspondencia a: Diego García-Huidobro Munita. dgarciah@med.puc.cl
\end{abstract}

\section{Introducción}

L as enfermedades causadas por el consumo de alimentos contaminados han aumentado en forma importante en los últimos años debido a los cambios en los sistemas de vida y los hábitos alimentarios de la población ${ }^{1,2}$. La Organización Mundial de la Salud define a las enfermedades de transmisión alimentaria como una enfermedad de naturaleza infecciosa o debida a toxinas, causada por agentes que ingresan al organismo a través del consumo de alimentos ${ }^{2}$. Se han descrito más de 250 agentes causales de enfermedades transmitidas por alimentos (ETA) entre los que se incluyen bacterias, virus, hongos, parásitos, priones, toxinas y metales, siendo más frecuentes las dos primeras ${ }^{3}$.

En la Región Metropolitana, el Servicio de Salud Metropolitano del Ambiente (SESMA) en coordinación con los servicios asistenciales de salud, inició en 1994 una vigilancia epidemiológica de los brotes de ETA. Actualmente es llevada a cabo por el equipo de epidemiólogos del Sub-Departamento de Epidemiología del Departamento de Salud Pública de la Secretaría Regional Ministerial (SEREMI) de Salud de la Región Metropolitana (RM).

Las cifras de notificación han aumentado globalmente. Durante el año 2008 en Estados Unidos de América (E.U.A.) se reportaron 1.034 brotes, afectando a 23.152 personas, causando 1.276 hospitalizaciones y 22 muertes $^{4}$. En nuestro país, durante el primer semestre del año 2011 fueron notificados 132 brotes de intoxicación alimentaria afectando a alrededor de 565 personas (Vollaire J, comunicación personal, Vigilancia Epidemiológica de ETA, Sub Departamento de Epidemiología, SEREMI de Salud RM.). En general, los principales agentes involucrados son Vibrio parahaemolyticus y Salmonella spp., siendo adquiridos principalmente en sitios externos a los hogares 5 .

El presente reporte presenta la descripción epidemiológica de un grave brote de ETA, la descripción clínica de múltiples casos que consultaron un mismo servicio de urgencia, y una revisión bibliográfica que integra los aspectos clínicos y de salud pública de este tipo de enfermedades.

\section{Descripción epidemiológica del brote}

Durante febrero de 2011 el Sub-Departamento de Epidemiología del Departamento de Salud Pública de la SEREMI de Salud de la RM., identificó un grave brote de ETA. La totalidad de los casos compartía el haber estado expuestos a alimentos de un restaurant de sushi del sector oriente de Santiago.

Epidemiológicamente se definió como caso a aquella persona que presentó al menos dos síntomas gastrointes- 
tinales luego de haber comido alimentos en el establecimiento involucrado, desde 36 horas previas al reporte del primer caso (caso índice). El informe epidemiológico del brote reportó un total de 38 casos, aislándose Salmonella Enteritidis en 16 de los 18 cultivos de deposiciones estudiados (Ulloa J, Sub Departamento de Epidemiología, SEREMI de Salud RM).

La curva epidémica del brote se presenta en la Figura 1, y la descripción socio-demográfica y clínica de todos los casos reportados se presentan en la Tabla 1. Destacaron dos casos que se manifestaron en pacientes embarazadas, donde una de ellas desarrolló un parto de pretérmino concomitantemente a la enfermedad. A pesar de haber presentado la misma sintomatología que el resto de los casos, su coprocultivo resultó negativo, y el recién nacido no presentó complicaciones.

Luego de que se notificaron los primeros tres casos del brote, se realizó una inspección al restaurant. En esta visita de inspección-fiscalizadora se detectaron deficiencias en la cadena de frío y contaminación cruzada de alimentos. Por esta razón, a las 5 horas y 25 minutos del reporte clínico, se cursó una prohibición de funcionamiento al establecimiento donde se consumieron los productos contaminados y se inició un sumario judicial.

\section{Descripción clínica de los casos}

Del total de casos reportados en este brote, en la Unidad de Emergencia de la Clínica UC San Carlos de Apoquindo, se atendió a 12 pacientes (31,6\% de los casos del brote estudiado): 11 adolescentes o adultos y 1 lactante de 1 año. Excluyendo los antecedentes del niño, los datos socio-demográficos y variables clínicas de los pacientes en la Unidad de Emergencia y de aquellos hospitalizados se resumen en la Tabla 2.

En general los intoxicados fueron personas jóvenes que presentaron síntomas similares al total de casos: diarrea sin elementos patológicos, dolor abdominal, náuseas, vómitos, fiebre, y gran compromiso del estado general. En la Unidad de Emergencia presentaron estabilidad hemodinámica, con taquicardia y fiebre, requiriendo en su totalidad la administración de hidratación endovenosa. La mayoría presentó múltiples consultas, y 45,5\% fueron diagnosticados correctamente en una segunda visita. Todos los casos a quienes se les tomó cultivo de deposiciones resultaron positivos para $S$. Enteritidis.

Los padres del lactante consultaron ya que su hijo presentó deposiciones diarreicas, asociadas a sangre, sin vómitos ni fiebre. En la evaluación de ingreso se constató buen estado general, sin fiebre ni signos de deshidratación. Dado que su madre era uno de los casos atendidos y refirió que su hijo había consumido escasa cantidad de sushi, se tomó un coprocultivo que resultó positivo para S. Enteritidis. Por la escasa gravedad clínica observada se manejó con hidratación oral ambulatoria asociado a

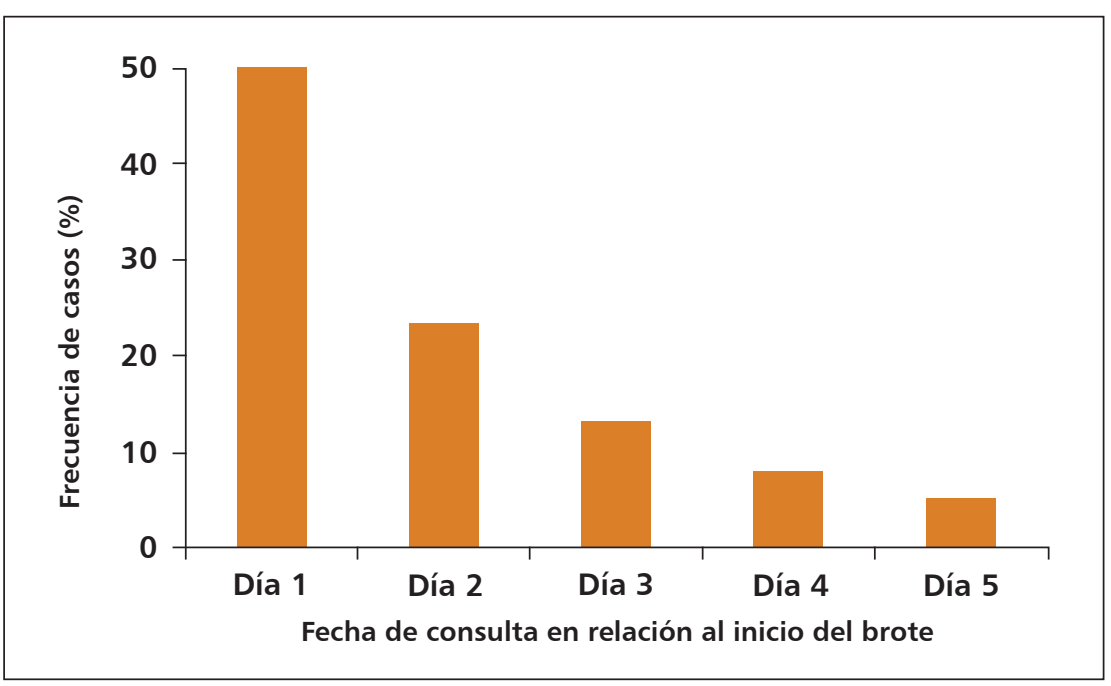

Figura 1. Curva epidémica de casos en el brote presentado.

Tabla 1. Descripción clínica de los 38 pacientes

reportados como casos intoxicados en el brote de Salmonella Enteritidis de Febrero 2011

\begin{tabular}{|lr|}
\hline $\begin{array}{l}\text { Sociodemográficas } \\
\text { Edad (años) }\end{array}$ & $28,4 \pm 9,7$ \\
Mujeres & $24(63,2 \%)$ \\
Atención médica & $33(86,8 \%)$ \\
Período de incubación (días) & $1,3 \pm 1,2$ \\
Manifestaciones clínicas & \\
Náuseas y vómitos & $27(71,0 \%)$ \\
Diarrea & $35(92,1 \%)$ \\
Dolor abdominal & $32(84,2 \%)$ \\
Fiebre & $34(89,5 \%)$ \\
Deposiciones con sangre & $3(7,8 \%)$ \\
Compromiso del estado general & $37(97,4 \%)$ \\
Deshidratación & $24(63,2 \%)$ \\
Hospitalización & $11(28,9 \%)$ \\
\hline
\end{tabular}

controles seriados en la Unidad de Emergencia. Durante el seguimiento se observó una buena evolución, sin requerirse el uso de antimicrobianos.

Un total de cinco pacientes fueron hospitalizados por deshidratación grave (41,7\%). Las variables sociodemográficas de los pacientes internados fueron similares a la de los pacientes consultantes a la Unidad de Emergencia. Durante su evolución, 60\% presentó hipotensión arterial sin ser necesario su trasladado a una Unidad de Paciente Crítico. En los exámenes de laboratorio destacó la leucocitosis y desviación izquierda, llegando a observarse 
Tabla 2. Descripción sociodemográfica y clínica de los 11 adolescentes y adultos consultantes en el Servicio de Urgencia de la Clínica UC San Carlos de Apoquindo

Sociodemográficas

Edad (años)

$26,4 \pm 11,7$

Mujeres

$6(54,5 \%)$

Variables clínicas en el Servicio de Urgencia

PAS / PAD ( $\mathrm{mmHg})$

$108 \pm 14 / 60 \pm 10$

Frecuencia cardíaca (latidos/min)

$101 \pm 18$

Temperatura (grados Celcius)

$37,9 \pm 0,8$

Número de consultas al servicio de urgencias

2 o más

$5(45,5 \%)$

$6(54,5 \%)$

Consulta donde se realizó diagnóstico

2

Exámenes de laboratorio en hospitalización

Leucocitos/mm3

$12.060 \pm 9.587$

Desviación a izquierda ( $\geq 10 \%$ baciliformes)

$3(60 \%)$

Proteína C reactiva $(\mathrm{mg} / \mathrm{dL})$

$14,7 \pm 9,3$

Creatininemia $(\mathrm{mg} / \mathrm{dL})$

$0,61 \pm 0,1$

Potasemia $(\mathrm{mEq} / \mathrm{L})$

$3,3 \pm 0,4$

Calcio corregido $(\mathrm{mg} / \mathrm{dL})$

$7,2 \pm 1,1$

Fosfemia (mg/dL)
A la totalidad de los pacientes adultos atendidos se les indicó tratamiento antimicrobiano, además de hidratación oral y antiespasmódicos. Quienes se manejaron exclusivamente en forma ambulatoria, recibieron ciprofloxacina 500 mg c/12 horas durante 5 días, y quienes se hospitalizaron recibieron inicialmente cefriaxona $1 \mathrm{~g}$ endovenoso al día, además de ciprofloxacina 500 mg c/12 horas vía oral hasta completar el tratamiento.

\section{Discusión}

Las ETA han aumentado su incidencia y afectan a más de 5.000 personas anualmente ${ }^{5}$, incrementando significativamente comparado al año 2000․ Este aumento del número de casos puede deberse a una mayor vigilancia epidemiológica por parte del Ministerio de Salud, además de los cambios en los hábitos de alimentación en las personas.

En general, las ETA son enfermedades que suelen ser leves y de tratamiento ambulatorio; sin embargo, ocasionalmente requieren hospitalización, y generan mortalidad. Durante el año 2007, en Chile se hospitalizaron 93 personas (1,7\%) y fallecieron dos pacientes, estimándose una letalidad de 0,04\% y mortalidad 0,0025 por 100.000 habitantes ${ }^{5}$.

Presentamos un grave brote de ETA ocasionado por $S$. Enteritidis. En este reporte, el número de casos fue seis veces mayor que el promedio de personas afectadas en cada brote durante los años 2006 y $2007^{5}$. Además, la presentación clínica de los pacientes fue de alta gravedad: 86,8\% de los casos notificados consultaron al médico y 28,9\% recibió tratamiento hospitalario, significativamente más que en otros brotes de nuestro país ${ }^{1,3,5} \mathrm{u}$ otros países ${ }^{4}$. Por el alto volumen de enfermos y la gravedad de las consecuencias, este brote fue catalogado como grave por las autoridades ministeriales.

Por otra parte, los pacientes adultos consultantes a la Unidad de Emergencia también presentaron gran deterioro clínico. La mayor parte tuvo fiebre, taquicardia y deshidratación, 54,5\% consultó en más de una oportunidad y todos requirieron hidratación endovenosa. Asimismo, de los pacientes hospitalizados, la mayoría presentó hipotensión arterial durante su estadía, leucocitosis con importante aumento del número de baciliformes, elevación de la proteína $\mathrm{C}$ reactiva y alteraciones bioquímicas compatibles con deshidratación grave.

Otra manifestación de la gravedad del brote fue el haber desencadenado el trabajo de parto prematuro en una de las mujeres embarazadas. A pesar de no haberse identificado $S$. Enteritidis en los cultivos de sangre o deposiciones, se podrían relacionar estos hechos ya que se han reportado casos de parto prematuro en pacientes embarazadas con infección por Salmonella no tífica ${ }^{7,8}$. 
Transmisión transplacentaria de la bacteria y los efectos de las endotoxinas explicarían esta consecuencia ${ }^{8}$. Por otra parte, la ausencia de complicaciones maternas y neonatales podrían deberse al avanzado estadio gestacional de la madre ${ }^{8}$. No se identificaron otras causas ginecoobstétricas que pudieran haber adelantado el inicio del trabajo de parto.

Razones que pudieran explicar la gravedad del brote son la virulencia de la bacteria identificada ${ }^{9,10}$, la carga bacteriana consumida ${ }^{11}$, y posible co-infección con otros agentes no identificados ${ }^{12,13}$. En un análisis de brotes durante 15 años en New York, se encontró agente causal sólo en $44 \%$ de las ETA asociadas a consumo de alimentos marinos, la mayoría fue de causa viral ${ }^{14}$. Ya que no se tomaron exámenes complementarios para determinar causas virales o toxinas en los pacientes, no es descartable que otros agentes infecciosos hayan estado involucrados en el brote reportado. Por otra parte, dado que la sensibilidad del coprocultivo para detectar parásitos en las ETA varía entre $37-74 \%{ }^{15}$, el hecho de que los resultados hayan sido negativos en los casos estudiados, tampoco descarta esta etiología.

Salmonella Enteritidis es un patógeno entérico responsable de $11 \%$ de los brotes de intoxicación alimentaria en nuestro país ${ }^{5}$, donde predominan los sub tipos B3 fagotipo 4 y B38 fagotipo $1^{16}$, o sub tipos $\mathrm{II}^{17}$. En general, tiene un período de incubación entre 8 horas y 3 días, provocando un cuadro clínico caracterizado por diarrea, fiebre y dolor abdominal, los que generalmente son autolimitados, con una duración máxima aproximada de 8 días. Frecuentemente las infecciones por este agente son de manejo ambulatorio, ya que rara vez se acompañan de complicaciones. Sin embargo, ese agente es el responsable de la mayoría de las hospitalizaciones por ETA ${ }^{4}$, puede generar complicaciones como disentería, deshidratación grave con insuficiencia renal, shock séptico, y eventualmente la muerte ${ }^{18}$.

El reservorio de este microorganismo es avícola y su transmisión es entérica, lo que involucra a alimentos de este origen para su transmisión ${ }^{19}$. Dado que el brote se originó en un restaurant de comida japonesa, la hipótesis para el origen del brote se relaciona a alteración en la cadena de frío y a contaminación cruzada de alimentos en el proceso de preparación del sushi. Otros agentes frecuentemente involucrados en brotes de ETA relacionados a comida marina son virus (calicivirus humano tipo norovirus, hepatitis A), bacterias (Vibrio spp, Shigella spp, Campylobacter spp, Clostridium botulinum, Listeria monocytogenes) y parásitos (Nematodes: Anisakis simplex; Trematodes; Protozoos: Giardia intestinalis, Cryptosporidium parvum; Cestodes; Fasciola) ${ }^{20,21}$. Estrategias para establecimientos y consumidores promovidas por el Ministerio de Salud para a la prevención de enfermedades transmitidas por alimentos marinos se mencionan en la Tabla 3.
Tabla 3. Estrategias específicas para la prevención de enfermedades

asociadas a alimentos marinos

Para los establecimientos:

1. Alimentos para el consumo crudo debieran congelarse al menos a una temperatura de $-35^{\circ} \mathrm{C}$ por 15 horas o al menos a una temperatura de $-20^{\circ} \mathrm{C}$ por 7 días

2. Descongelar los productos 14 horas previas a la ingesta

3. Descongelar alimentos en el refrigerador y no a temperatura ambiental

4. No mantener pescado en el refrigerador por más de tres días

5. Una vez removidos del refrigerador, consumir alimentos antes de tres horas

6. Cocer bien los alimentos antes de servirlos

7. Separar los alimentos cocidos de los crudos, asimismo los utensilios usados para su preparación, para evitar una contaminación cruzada

Para los consumidores:

1. Consumir alimentos completamente cocidos

2. Comer en establecimientos donde se pueda observar la manipulación de los alimentos que serán consumidos

3. Recordar que el limón no cuece los alimentos, sino que es sólo un condimento

Dado que la mayoría de las ETA ocurren en establecimientos fuera del hogar ${ }^{4,5}$ es fundamental un adecuado sistema de monitoreo. La vigilancia de ETA en la RM contempla tres unidades: vigilancia de morbilidad a cargo del Sub-Departamento de Epidemiología, vigilancia de laboratorio a cargo del Sub-Departamento de Vigilancia Ambiental, ambos dependientes del Departamento de Salud Pública de la SEREMI de Salud de la RM y la tercera unidad la constituye la vigilancia epidemiológica de laboratorio para cepas específicas que realiza el Instituto de Salud Pública de Chile.

Para activar oportunamente este sistema de monitorización, seguimiento y acción, los médicos debemos notificar inmediatamente al Sub-Departamento de Epidemiología la sospecha de un brote de ETA. Así, el equipo de epidemiólogos podrá realizar la investigación del brote, obtener muestras, y tomar las medidas necesarias para el control de la intoxicación. Habitualmente sólo se procuran muestras de los alimentos específicamente involucrados en un brote. Este brote fue detectado 32 horas después de la ingesta del primer caso; no se obtuvieron muestras de alimentos por no tener acceso a éstos.

A pesar de la relevancia de la notificación precoz, destaca que $45,5 \%$ de los pacientes que consultaron en la Unidad de Emergencia no se diagnosticaron en una primera consulta, retrasando la activación del sistema de monitorización sanitaria y sobrecargando la demanda asistencial. Se han reportado varias causas para la subnotificación de brotes por enfermedades gastrointestinales por médicos en los servicios de urgencia ${ }^{21}$. Entre éstas se encuentran el no considerar las ETA dentro del diagnóstico diferencial, no solicitar exámenes de laboratorio, 
consultas por síntomas leves que independiente del diagnóstico no requerirán tratamiento específico, y la ausencia de reporte electrónico sistemático en los motivos de consulta y diagnósticos de los pacientes. En nuestro país se ha descrito que los médicos, especialmente en los servicios de urgencia, no se sienten motivados a efectuar la notificación restándole prioridad dentro de sus tareas asistenciales $^{1}$. A partir de estas observaciones, se han desarrollado sistemas de vigilancia computacionales basados en registros clínicos electrónicos. Estos instrumentos realizan reportes automáticos de acuerdo a los motivos de consulta médica y a los diagnósticos realizados ${ }^{23}$. Mejorar los sistemas de alerta permitirá detectar precozmente el surgimiento de brotes epidemiológicos para así evitar las consecuencias de estas enfermedades. Mientras logremos implementar estos sistemas de monitoreo se hace imprescindible agudizar la práctica clínica y considerar el diagnóstico diferencial de las ETA dentro de los cuadros de síndrome diarreico agudo ${ }^{24}$.

Por los resultados de la visita evaluadora se cursó una prohibición de funcionamiento y un sumario judicial, que hasta la fecha, aún se encuentra en proceso (octubre 2011). Es relevante mencionar que el sumario se cursa por los déficits encontrados, independiente de la aparición de un brote, a menos que se tengan muestras de los alimentos contaminados. El tiempo de demora en la re-apertura de los establecimientos depende del tiempo que tarden éstos en mejorar las deficiencias en infraestructura o procesos detectadas en la inspección. En el caso reportado, esta prohibición se mantuvo por tres días hasta que en una nueva visita se demostró la mejoría de la situación detectada previamente.

En este artículo, reportamos un grave brote causado por
S. Enteritidis. Dado la importancia de las consecuencias individuales y poblacionales de las enfermedades transmitidas por alimentos, es imprescindible que los médicos de los servicios de urgencia y de atención primaria comuniquemos oportunamente a la SEREMI de Salud la sospecha de un brote de intoxicación alimentaria para activar el sistema de vigilancia y prevenir el contagio de esta enfermedad a otras personas. De esta forma contribuiremos a reducir globalmente la morbi-mortalidad asociada a estos brotes y a mejorar la salud de todos.

\section{Resumen}

Introducción: Las enfermedades transmitidas por alimentos (ETA) han aumentado considerablemente. Objetivo: Reportar un grave brote de ETA destacando la importancia de la notificación precoz para la activación del sistema de vigilancia epidemiológica. Resultados: Durante febrero de 2011 se observó un brote de Salmonella Enteritidis. Un 31,6\% de los casos fueron atendidos en un mismo servicio de urgencia, donde todos requirieron la administración de fluidos endovenosos y 41,7\% fueron hospitalizados por deshidratación grave. El 45,5\% de los casos necesitó de una segunda consulta para ser diagnosticados correctamente. Discusión: La identificación de pacientes integrantes de un brote de ETA es difícil en los servicios de urgencia y los médicos sub-reportan los casos, retrasando al sistema de vigilancia epidemiológica. Conclusiones: Junto con brindar un adecuado tratamiento, los médicos deben estar capacitados para reconocer las ETA y comunicar tempranamente la sospecha de un brote para prevenir nuevos casos en la comunidad.

\section{Referencias}

1.- Prado V, Solari V, Álvarez I, Arellano C, Vidal R, Carreño M, et al. Situación epidemiológica de las enfermedades transmitidas por alimentos en Santiago de Chile: Período 1999-2000. Rev Med Chile 2002; 130: 495-501.

2- Organización Mundial de la Salud. Food Safety and Foodborne Illness. Fact Sheet $\mathrm{N}^{\circ}$ 237. 2007. Disponible en: http://www.who.int/ mediacentre/factsheets/fs237/en/, accedido el 24 de octubre de 2011.

3.- Olea A. Las enfermedades transmitidas por alimentos: un fenómeno frecuente de magnitud real desconocida. El Vigía: Boletín de Vigilancia de Salud Pública. 2007; 10: 37-42.

4.- Centers for Disease Control and Prevention (CDC). Surveillance for foodborne disease outbreaks-United States, 2008. MMWR Morb Mortal Wkly Rep 2011; 60: 1197-202.

5.- Departamento de Epidemiología, División de Planificación Sanitaria, Ministerio de Salud. Situación Epidemiológica de las Enfermedades Transmitidas por Alimentos en Chile. 2007. Disponible en: http://epi.minsal.cl/epi/html/ presenta/VETA2007.pdf, accedido el 24 de Octubre de 2011.

6.- Chanqueo L, García P, León E, Blu A. Evaluación de la tinción de Hucker para la búsqueda rutinaria de Campylobacter sp en el estudio de un síndrome diarreico agudo. Rev Chil Infectol 2005; 22: 242-6.

7.- Roll C, Schmid E N, Menken U, Hanssler L. Fatal Salmonella enteritidis sepsis acquired prenatally in a premature infant. Obstet Gynecol 1996; 88: 692-3.

8.- Schloesser R L, Schaefer V, Groll A H. Fatal transplacental infection with non- typhoidal Salmonella. Scand J Infect Dis 2004; 36: 773-4.

9.- Foley SL, Lynne AM. Food animal-associated Salmonella challenges: pathogenicity and antimicrobial resistance. J Anim Sci 2008; 86: E173-87.
10.- Alexandre M, Pozo C, González V, Martínez M C, Prat S, Fernández A, et al. Detección de Salmonella enteritidis en muestras de productos avícolas de consumo humano en la Región Metropolitana. Rev Med Chile 2000; 128: 1075-83.

11. Teunis PF, Kasuga F, Fazil A, Ogden I D, Rotariu O, Strachan N J. Dose-response modeling of Salmonella using outbreak data. Int J Food Microbiol 2010; 144: 243-9.

12.- Sabota J M, Hoppes W L, Ziegler J R, DuPont H, Mathewson J, Rutecki G W. A new variant of food poisoning: enteroinvasive Klebsiella pneumoniae and Escherichia coli sepsis from a contaminated hamburger. Am J Gastroenterol 1998; 93: 118-9.

13.- Duc le H, Dong T C, Logan N A, Sutherland A D, Taylor J, Cutting S M. Cases of emesis associated with bacterial contamination of an infant breakfast cereal product. Int J Food Microbiol 2005; 102: 245-51. 
14.- Wallace B J, Guzewich J J. Cambridge M, Altekruse S, Morse D L. Seafood-associated disease outbreaks in New York, 1980-1994. Am J Prev Med 1999; 17: 48-54.

15.- Abubakar I, Irvine L, Aldus CF, Wyatt GM, Fordham R, Schelenz S, et al. A systematic review of the clinical, public health and cost-effectiveness of rapid diagnostic tests for the detection and identification of bacterial intestinal pathogens in faeces and food. Health Technol Assess 2007; 11: 1-216.

16.- Fernández J, Fica A, Ebensperger G, Calfullan H, Prat S, Fernández A, et al. Analysis of molecular epidemiology of Chilean Salmonella enterica serotype Enteritidis isolates by pulsed-field gel electrophoresis and bacteriophage typing. J Clin Microbiol 2003 41: 1617-22.

17.- Ríos M, Araya P, Fernández A, Tognarelli J,
Hormazábal J C, Fernández J. Subtipificación molecular de Salmonella enterica serotipo Enteritidis en el período post epidémico. Rev Med Chile 2009; 137: 71-5.

18.- Fica A, Caorsi B, Piemonte P. Dysenteric syndrome, acute renal failure and lethal septic shock associated to Salmonella enteritidis infection. Report of 3 cases. Rev Med Chile 1997; 125: 1055-82.

19.- Fica A, Alexandre M, Prat S, Fernández A, Fernández J, Heitmann I. Cambios epidemiológicos de las salmonellosis en Chile. Desde Salmonella typhi a Salmonella enteritidis. Rev Chil Infectol 2001; 18: 85-93.

20.- Butt A, Aldridge K, Sanders C. Infections related to the ingestion of seafood. Part I: viral and bacterial infections. Lancet Infect Dis 2004; 4: 201-12.
21. Iwamoto M, Ayers T, Mahon B E, Swerdlow D L. Epidemiology of seafood-associated infections in the United States. Clin Microbiol Rev 2010; 23: 399-411.

22.- Balter S, Weiss D, Hnson H, Reddy V, Das D, Heffernan R. Three years of emergency department gastrointestinal syndromic surveillance in New York City: What have we found? MMWR Morb Mortal Wkly Rep 2005; 54 (Suppl); 175-80.

23.- Buckeridge D L, Okhmatovskaia A, Tu S, O’Connor M, Nyulas C, Musen MA. Predicting outbreak detection in public health surveillance: quantitative analysis to enable evidence-based method selection. AMIA Annu Symp Proc 2008: 76-80.

24.- Fica A. Manejo ambulatorio del síndrome diarreico agudo en adultos. Rev Chil Infectol 2001; 18: 108-26. 